\title{
Aplicación de las Tecnologías de la Información y la Comunicación en los procesos de innovación empresarial. Revisión de la literatura
}

Application of Information and Communication Technologies in business innovation processes.

Literature Review

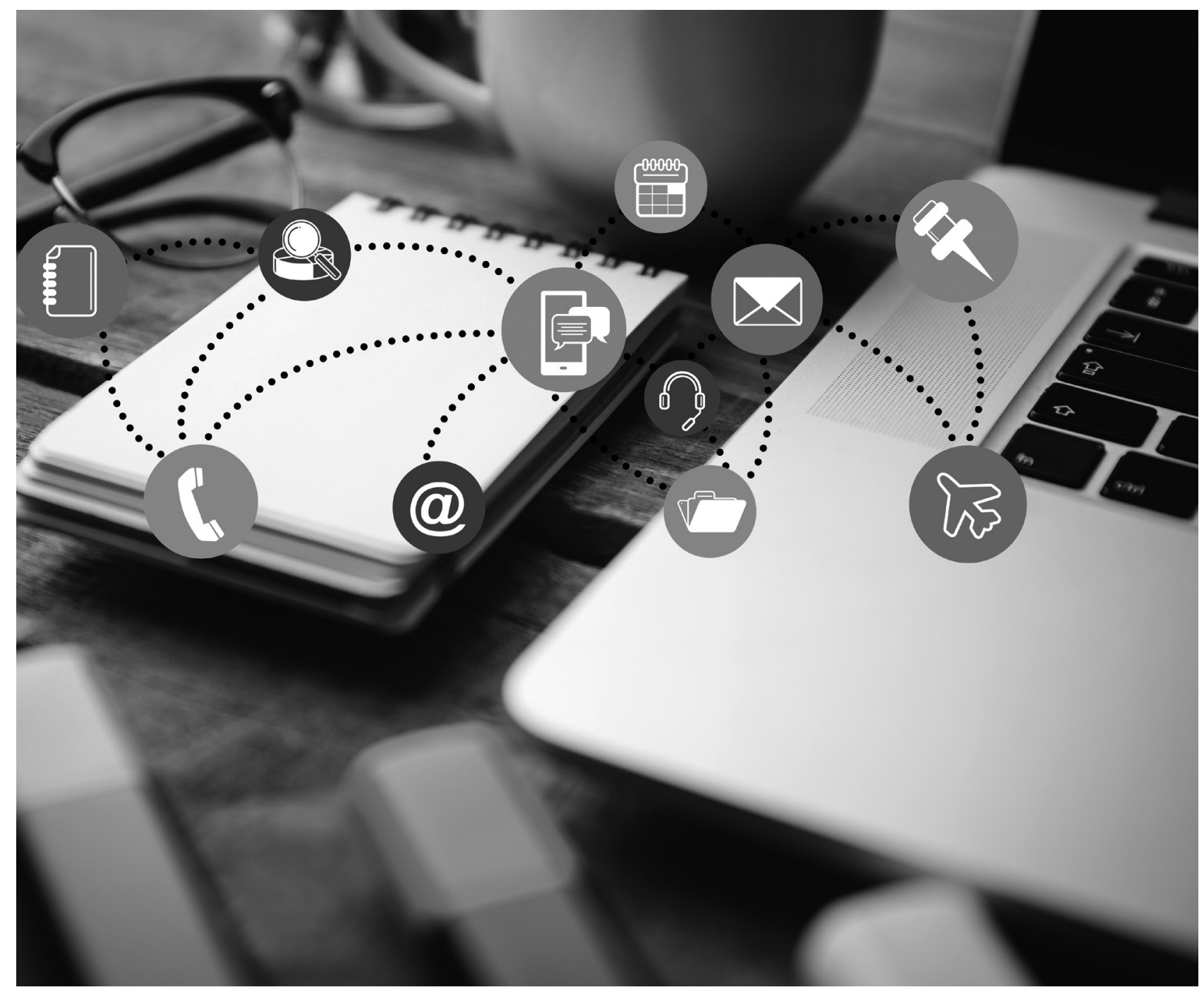




\title{
Aplicación de las Tecnologías de la Información y la Comunicación en los procesos de innovación empresarial. Revisión de la literatura ${ }^{1}$
}

\section{Application of Information and Communication Technologies in business innovation processes. Literature Review}

\section{Omar Alexander León García ${ }^{2}$, Edna Nayibe Palma Hernández ${ }^{3}$}

Artículo recibido en agosto de 2017; artículo aceptado en septiembre de 2017.

Este artículo puede compartirse bajo la Licencia Creative Commons Atribución-No Comercial-Compartir lgual 2.0 Genérica

y se referencia usando el siguiente formato: León, O., Palma, E. (2018). Aplicación de las Tecnologías de Información y comunicación en los procesos de innovación empresarial. Revisión de la literatura. I+D Revista de Investigaciones, 11(1), $144-152$.

DOI: https://doi.org/10.33304/revinv.v11n1-2018012

\begin{abstract}
Resumen
El objetivo principal de este estudio es identificar la producción académica y científica en torno a la relación existente entre el uso de las Tecnologías de la Información y la Comunicación (TIC) y la innovación empresarial. En este sentido, este artículo analiza las investigaciones empíricas que han abordado esta temática durante el periodo 2007 - 2016, evidenciando que la mayoría de los estudios muestran esta relación desde el punto de vista del uso que hacen las empresas de este recurso y como esto afecta los procesos de innovación empresarial. El documento registra las principales definiciones y variables utilizadas en los estudios, identifica la manera como las TIC pueden mejorar los procesos de innovación en las empresas y, finalmente, plantea las posibles líneas de investigación futuras.
\end{abstract}

Palabras clave: TIC, innovación, innovación empresarial, estrategia de innovación, innovación de procesos, pyme, cadena de valor.

\begin{abstract}
The main objective of this study is to identify academic and scientific production about the relationship between the use of information and communication technologies (ICT) and business innovation. In this sense, this article analyzes the empirical researches that have addressed this issue during the 2007-2016 period, evidencing that most studies show this relationship from the point of view of the use that companies make of this resource and how it affects the business innovation processes. The document records the main definitions and variables used in the studies and identifies how
\end{abstract}

1. Artículo de investigación, enfoque cualitativo, resultado de un proyecto de investigación en curso, perteneciente al área de Ingeniería, desarrollado en el Grupo de Investigación en Ingeniería de Sistemas - GIIS, financiado por la Unipanamericana Compensar Institución Universitaria y la empresa Draco Servicios de la ciudad de Bogotá (Colombia). Dirección: Avenida (calle) 32 n. ${ }^{\circ} 17-30$ y Carrera 7 H bis n. ${ }^{\circ} 159-23$ (respectivamente). Fecha de inicio: 18 de enero de 2017.

2. Ingeniero de Producción Universidad EAN, PhD en Ingeniería Mondragon Unibertsitatea, España. Investigador y Docente vinculado al Grupo de investigación GIIS de la Facultad de Ingeniería de Unipanamericana Compensar Institución Universitaria. Dirección: Avenida (calle) 32 n. 17 - 30. PBX: 33806 66. Bogotá (Colombia). ORCID ID: https://orcid.org/0000-0001-5554-8215. Correo electrónico institucional: omarleon@unipanamericana.edu.co.

3. Estudiante del programa de Ingeniería de Sistemas de la Facultad de Ingeniería de Unipanamericana Compensar Institución Universitaria. Grupo de Investigación GIIS de la Facultad de Ingeniería de Unipanamericana Compensar Institución Universitaria. Dirección: Avenida (calle) 32 n. 17 - 30. PBX: 338 06 66. Bogotá (Colombia). ORCID ID: https://orcid.org/0000-0003-4330172X. Correo electrónico institucional: epalma@unipanamericana.edu.co. 
Omar Alexander León García, Edna Nayibe Palma Hernández

Aplicación de las Tecnologías de la Información y la comunicación en los procesos de innovación empresarial. Revisión de la literatura

ICT can improve innovation processes in companies and finally considers possible future lines of research.

Keywords: ICT, innovation, company innovation, innovation strategy, process innovation, SMEs, value chain.

\section{Introducción}

Una parte importante de la estrategia de innovación de las pyme es el diseño de nuevos productos $y$, en particular, la determinación del nivel de calidad que debe alcanzarse antes de su introducción al mercado (Dawid, \& Reimann, 2011). De hecho, una empresa en un mercado de competencia imperfecta tiene incentivos para mejorar constantemente la calidad de sus productos con el fin de evitar la vulnerabilidad a los posibles competidores. Esta mejora de la calidad puede requerir cierta diversificación de su base tecnológica (Garcia, 2007). Es decir, las empresas tienen que "abarcar sus actividades innovadoras sobre más de una tecnología" (Breschi, Lissoni \& Montobbio, 2007) con la idea de que este impacto se evidencie en alcanzar un mercado más amplio.

La innovación se debe convertir en procesos que generan ideas, desarrollan proyectos que permitan realizar un proceso de prueba-error en el mercado, hasta convertirlas en una actividad rutinaria dentro de la empresa y concebirla como una ventaja competitiva (Barreto, 2017). El concepto de innovación es, por tanto, complejo y difuso; sin embargo, parece existir un denominador común a todas sus definiciones: el concepto de novedad (Fonseca, 2013).

La innovación es el proceso de integración de la tecnología existente para crear o mejorar un producto, un proceso o un sistema (Riascos, Aguilera, \& Solano, 2015). A la adopción de una idea o comportamiento nuevo en una organización también se le considera innovación (Damanpour \& Gopalakrishnan, 1998).

La innovación no es resultado de una investigación sistemática sino de la exploración a partir de la experiencia de las empresas, que en busca de ventajas respecto de sus competidores apoyan al proceso de innovación viéndolo como un desarrollo (Espinosa et al., 2016).

En una amplia gama de industrias, la innovación es la base de la capacidad de las empresas para mantener una ventaja competitiva (Miller, Fern \& Cardinal, 2007), debido, entre otras cosas, a que los elementos clave que indican la evolución de una industria para convertirse en una más global, incluyen la estandarización de los productos y servicios debido a la homogeneización de los gustos de los consumidores, así como el desarrollo de las economías de escala mundial en la fabricación y en investigación y desarrollo (I+D) (Wiersema \& Bowen, 2008).
De igual manera, el concepto de capacidad de innovación está altamente asociado a la capacidad de I+D de la empresa, pero, sin lugar a dudas, esta ha de ser complementada por la gestión necesaria que debe desarrollarse en otras áreas funcionales de la organización (López \& Robledo, 2014).

Es común que después de alcanzado el nuevo desarrollo no se continúe haciendo investigación, sobre todo por problemas económicos y porque no es habitual hacer proyectos; en consecuencia, es común que se realice una contratación de trabajadores sin mayor cualificación, que no se participe en ningún tipo de ferias y no se plantee establecer contactos con instituciones estatales como clientes de sus productos (Espinosa et al., 2016).

De otro lado, las TIC son el conjunto de sistemas y productos que captan la información del entorno, la almacenan, la procesan, la comunican y la hacen inteligible a las personas, y la transmiten a través de la interconexión de equipos que facilitan la construcción de redes (Riascos, Aguilera \& Solano, 2015). Las TIC están constituidas por las inversiones que la empresa ha realizado en equipos de computación, software y medios de comunicación (Gálvez, De Lema \& García, 2012). El uso de estas tecnologías ha provocado una importante transformación en todos los niveles de la organización, siendo la actividad de innovación una de las beneficiadas (Soto, Senra, \& Neira, 2009).

Es sabido que las TIC acrecientan la habilidad de mantener buenas y mejores relaciones tanto dentro como fuera de las empresas (McDonough, Kahnb \& Barczaka, 2001; Rothwell, 1994), permiten mejorar la colaboración entre los empleados (Smith \& Blanck, 2002) y las relaciones con agentes externos (Kahn, 2001). Estas características hacen que estas tecnologías se conviertan en un factor importante para las empresas que desean mantenerse en un mercado cada vez más competitivo (Argüello, 2015).

Las TIC constituyen uno de los medios más adecuados para aproximarse al entorno y desarrollar un profundo conocimiento sobre los diferentes agentes que lo conforman. De esta forma, actuando como una fuente de obtención y generación de información, permiten que la empresa acceda de forma sencilla, rápida y económica a una gran cantidad de información relevante y actual de clientes y competidores (Jimenez \& Torrent, 2009; Soto \& Rios, 2017).

Las TIC hacen posible un mayor trabajo en red, ofreciendo la información suficiente y oportuna para tomar decisiones y responder a las exigencias del mercado, a la vez que se 
desarrollan estrategias para la aplicación de estas tecnologías; por esta razón, las empresas desarrollan su actividad en un nuevo "entorno digital" que les permite implementar el comercio electrónico de forma natural, de manera que deje de llamarse "electrónico" y pase a denominarse simplemente "comercio", puesto que lo importante no es si se hace por Internet o por otras vías (Martínez \& Castillo, 2014).

El uso de estas herramientas tecnológicas en los diferentes procesos de la empresa es un catalizador de innovación que incrementa la competitividad, siempre que dichas herramientas estén enfocadas en las necesidades reales de la empresa y alineadas con la estrategia del negocio, ya que, por ejemplo, fortalecen la comunicación entre los empleados y permiten consolidar relaciones de confianza, y construir una actitud de colaboración en las personas de los diferentes niveles de la estructura (Mosquera, Rojas \& Grillo, 2015).

Finalmente, debido a que la ciencia ha avanzado en sus prácticas para ayudar a quienes, por medio de las TIC, pretenden mejorar los procesos de innovación en las empresas, se está estimulando el acceso y apoyo a través de fondos para emprendedores, formación de clústeres, capacitación, entre otros (Graells, 2000). A pesar de esto hay que ver cuánto falta en materia de adopción de TIC en las empresas, ya que, aunque este sector es cada vez más atractivo y competitivo en un mundo que demanda calidad, servicio y agilidad en los procesos de prestación de servicio o entrega de productos, aún falta bastante para que las pyme utilicen las TIC más profundamente en sus procesos (Almenara, 2004). En este sentido, se evidencia que el análisis de la relación entre las TIC y las estrategias de innovación es un área de considerable interés tanto para la comunidad científica como para el mundo empresarial, que ven la importancia y justifican este tipo de investigación en el alto impacto que tiene sobre las pequeñas y medianas empresas (Hoyos \& Valencia, 2012).

De acuerdo con lo anterior, es evidente la necesidad de realizar una revisión de la literatura existente con la idea de reconocer los aportes científicos de la temática, identificar variables de medición utilizadas en los estudios prácticos, y reconocer las mejoras que estas tecnologías brindan a las pyme.

\section{Metodología}

Este estudio es de tipo descriptivo, se ha elaborado a partir de la recopilación de cuarenta artículos científicos que se han desarrollado en torno al impacto de las TIC en los procesos de innovación de las pyme en el periodo comprendido entre 2007 y 2017.

Para la recolección de la información se llevó a cabo una búsqueda y clasificación de revistas y artículos científicos publicados y ubicados en las bases de datos Jstor, Dialnet, Redalyc, Scielo, Latindex, Google académico y ProQuest; igualmente, se consultó el portal DOAJ, que es un directorio multidisciplinar de revistas de acceso abierto con enlace a las páginas web que ofrecen los textos completos de los artículos.

Para la elaboración del presente estudio se tuvo en cuenta la metodología sugerida por Hoyos (1999) para el desarrollo de estado del arte, la cual sugiere contextualizar, clasificar y analizar los artículos seleccionados para identificar sus resultados de acuerdo a las categorías reconocidas (TIC, procesos de innovación, empresa, tecnología).

\section{Resultados}

Los resultados, de acuerdo con la revisión de la literatura, permitieron establecer los efectos de la cooperación y el uso de las TIC sobre el éxito de las innovaciones en los procesos de las pyme.

\section{Concepto de innovación}

En los textos analizados se identifican varias definiciones de lo que se entiende por innovación. Desde los trabajos iniciales de este tema se concebía la innovación como la introducción de un nuevo bien o método, cambio en la calidad, apertura de un nuevo mercado, creación de una nueva organización (Schumpeter, 1934; Tushman \& Nadler, 1986) o como la adopción de una idea o comportamiento nuevo en una organización (Damanpour \& Gopalakrishnan, 1998). Acorde con esto, el Manual de Oslo la define como "la introducción de un nuevo, o significativamente mejorado producto (bien o servicio), de un proceso, de un nuevo método de comercialización o de un nuevo método organizativo, en las prácticas internas de la empresa, la organización del lugar de trabajo o las relaciones externas" ([OECD], 2005).

Conceptos actuales de innovación la definen como una idea transformada en algo vendido o usado (Salvador, Brand \& Salazar, 2011), lo que implica que una mejora del producto y una reducción de sus costes, que se verá reflejado en que la empresa aumentará su beneficio y su cuota de mercado (Gálvez, De Lema \& García, 2012). La innovación, en sentido económico, consisteen la consolidación de un nuevo producto, proceso o sistema mejorado. También es reconocida como la introducción de algo nuevo con el propósito de generar un cambio grande, radical. La innovación consiste en producir, asimilar y explotar con éxito la novedad en los ámbitos económico y social (Triana, Medina \& Rodríguez, 2013).

Otros autores la definen como la entrada en el mercado de un producto o proceso nuevo o significativamente 
mejorado o el desarrollo de nuevas técnicas de organización y comercialización (Cano \& Baena, 2013). Finalmente, la innovación es el proceso de integración de la tecnología existente para crear o mejorar un producto, un proceso o un sistema (Riascos, Aguilera \& Solano, 2015).

En resumen, el examen de la bibliografía existente mostró que la literatura ofrece un conjunto variado de definiciones y términos relativos al concepto de innovación, sin importar las características que cada autor enfatiza. Se observa que estas definiciones en común concuerdan con que la innovación transforma, desde diferentes puntos de vista, una organización, considerándose como un factor inicial para la construcción del éxito empresarial.

Las variables identificadas en los estudios (ver Tabla 1) están relacionadas con la innovación en procesos y mercados a nivel global. El uso de las TIC muestra que su impacto se ve reflejado en procesos productivos más eficientes y la comercialización en nuevos mercados (Fonseca, 2013; Marulanda \& López, 2013).

Una vez la empresa incluye un nuevo producto en nuevo mercado, se habla de que estas tecnologías han favorecido un proceso de diversificación empresarial, lo que ha permitido generar nuevas líneas de negocio (Castellanos, Loaiza \& Cuesta, 2016; García, López \& Epalde, 2015).

Los resultados permiten mostrar la importancia de hacer un mejor y más intensivo uso de las TIC con la idea de conseguir mejores resultados empresariales a través de los procesos de innovación.

Tabla 1

Variables utilizadas en la selección de los artículos publicados para innovación

\begin{tabular}{|c|c|c|c|}
\hline Variable & Indicador & Resultado & Referencia \\
\hline $\begin{array}{l}\text { Innovación de producto } \\
\text { y servicio }\end{array}$ & $\begin{array}{l}\text { Uso de TIC, generación, } \\
\text { cambios o mejoras de } \\
\text { productos, innovación de } \\
\text { producto. }\end{array}$ & $\begin{array}{l}\text { Influye directamente } \\
\text { en el producto. }\end{array}$ & $\begin{array}{l}\text { (Jimenez \& Torrent, 2009, } \\
\text { Vilaseca et al., 2007) (Jiménez, } \\
\text { Martínez \& Barba, 2009) } \\
\text { (Fonseca, 2013; Marulanda, } \\
\text { Giraldo \& López, 2014). }\end{array}$ \\
\hline $\begin{array}{l}\text { Innovación en productos } \\
\text { y procesos }\end{array}$ & $\begin{array}{l}\text { Mejoras o cambios en los } \\
\text { procesos productivos de la } \\
\text { organización a través de la } \\
\text { gestión del conocimiento e } \\
\text { internacionalización, mediante } \\
\text { la apropiación de las TIC }\end{array}$ & $\begin{array}{l}\text { Influye en las } \\
\text { necesidades directas } \\
\text { de los procesos. }\end{array}$ & $\begin{array}{l}\text { (Fonseca, 2013; Marulanda \& } \\
\text { López, 2013) (Cano \& Baena, } \\
\text { 2013; Alderete \& Gutiérrez, } \\
\text { 2012; Mosquera, Rojas \& Grillo, } \\
\text { 2015). }\end{array}$ \\
\hline Innovación de mercados & $\begin{array}{l}\text { TIC como un elemento que } \\
\text { puede conducir a la empresas a } \\
\text { mejorar su competitividad. }\end{array}$ & $\begin{array}{l}\text { Comercialización de } \\
\text { productos en nuevos } \\
\text { mercados. }\end{array}$ & $\begin{array}{l}\text { (Segovia, Hernández, \& Ramírez, } \\
\text { 2013; Gálvez, De Lema, \& García, } \\
\text { 2012). }\end{array}$ \\
\hline Innovación global & $\begin{array}{l}\text { Capacidad innovadora de la } \\
\text { organización. }\end{array}$ & $\begin{array}{l}\text { Comercialización y } \\
\text { ventas de productos } \\
\text { de alta tecnología. }\end{array}$ & $\begin{array}{l}\text { (Avendaxño, 2012; Acevedo, } \\
\text { Moreno \& Sánchez, 2013; } \\
\text { Gálvez, 2014). }\end{array}$ \\
\hline Diversificación & $\begin{array}{l}\text { Tipo y grado de diversificación } \\
\text { empresarial. }\end{array}$ & $\begin{array}{l}\text { Influye en la creación } \\
\text { de nuevos productos y } \\
\text { servicios para llegar a } \\
\text { nuevos mercados. }\end{array}$ & $\begin{array}{l}\text { (Martin \& Omrani, 2014; } \\
\text { Castellanos, Loaiza, \& Cuesta, } \\
\text { 2016; Arcila, Calderín \& } \\
\text { Aguaded, 2015). }\end{array}$ \\
\hline
\end{tabular}

Fuente: Autores.

\section{Las TIC mejoran los procesos de innovación en las empresas}

Siendo incuestionable que estas tecnologías tienen una alta repercusión en la economía moderna (Zhang, Van Donk \& Van der Vaart, 2011), su amplio desarrollo ha afectado la dinámica de la competencia empresarial, la globalización, las relaciones con los clientes y la innovación tecnológica, revolucionando el proceso de compra y venta de productos y servicios (Kim, Lee \& Lee, 2013). El avance en las TIC ha dado paso a un nuevo género de oportunidades de negocio que permite ampliar el horizonte de las empresas para explorar nuevos modelos de negocio. Este nuevo siglo presenta una fuerte propensión para que las empresas adopten estas tecnologías (como el Internet) como medio para una nueva conducta empresarial (Sharma, 2013).

Las TIC mejoran el desempeño de los procesos de cooperación interorganizacional, lo quefavorece los procesos de innovación empresarial, permitiendo la integración entre la mejora y el 
desempeño de los procesos estratégicos de la compañía y las redes informáticas (Jones \&Morero, 2014), aunque es necesario incorporar los procesos de innovación mediante la gestión de conocimiento, ya que permiten identificar, compartir y aplicar conocimiento como avance importante para el sector de la ciencia y la tecnología (Hernández, Marulanda \& López, 2014).

Gracias a las TIC existen oportunidades que fortalecen los mercados para que las empresas mejoren la relación con los clientes a través de los diversos canales de comunicación (Osorio, Londoño \& López, 2016). Además, es muy importante el nivel de integración que tienen las TIC con los procesos administrativos y operativos de la organización, ya que permiten la entrega oportuna de productos y servicios (Castellanos, Loaiza \& Cuesta, 2016).

El uso adecuado de las TIC aumenta la productividad, en especial para las pyme, ampliando la posibilidad de nuevos negocios.

Estas ventajas se han desarrollado y comprobado en empresas de mayor tamaño, por lo que deben extenderse a las pyme en tanto permiten registrar la información contable y de gestión administrativa de forma eficiente (Urquía \& Pérez, 2012).

Las organizaciones de hoy suponen que las TIC son una forma de luchar contra la competencia mediante la mejora de la productividad y la calidad de las operaciones (Devaraj \& Kohli, 2003), ya que sus innovaciones han ofrecido oportunidades para mejorar sus procesos y desarrollar nuevos modelos de negocio (Kim, Lee \& Lee, 2013).

\section{Tabla 2}

\section{Tecnologías de la información en la cadena de valor}

\begin{tabular}{|c|c|c|}
\hline $\begin{array}{l}\text { Cadena de } \\
\text { valor }\end{array}$ & procesos & Herramientas TIC \\
\hline \multirow[t]{3}{*}{$\begin{array}{l}\text { Cadena } \\
\text { primaria }\end{array}$} & Diseño & $\begin{array}{l}\text { Diseño asistido, simuladores, inteligencia competitiva, test de productos, } \\
\text { herramientas Brainstorming. }\end{array}$ \\
\hline & Operaciones & $\begin{array}{l}\text { Supply chain management (SCM), mercados digitales, Intercambio de } \\
\text { información integrada, automatización de fábricas, impresión 3D, gestión } \\
\text { de redes, Radio Frequency IDentification (RFID), Global Positioning System } \\
\text { (GPS). }\end{array}$ \\
\hline & Cliente & $\begin{array}{l}\text { Catálogos digitales, información de productos, compra on-line, Customer } \\
\text { Relationship Management (CRM), personalización de productos, e-business, } \\
\text { e-marketing, M-mail, web social, inteligencia de mercados. }\end{array}$ \\
\hline \multirow[t]{4}{*}{$\begin{array}{l}\text { Cadena } \\
\text { secundaria }\end{array}$} & Administración & $\begin{array}{l}\text { Ofimática, Comunicaciones, Herramientas de colaboración, Enterprise } \\
\text { Resource Planning (ERP). }\end{array}$ \\
\hline & $\begin{array}{l}\text { Información de } \\
\text { gestión }\end{array}$ & $\begin{array}{l}\text { Intranet, Portal empresarial, Plataformas de gestión del conocimiento, web } \\
\text { social. }\end{array}$ \\
\hline & Recursos humanos & $\begin{array}{l}\text { Teletrabajo, e-learning, gestión de competencias, evaluadores de } \\
\text { productividad }\end{array}$ \\
\hline & $\begin{array}{l}\text { Contabilidad y } \\
\text { finanzas }\end{array}$ & $\begin{array}{l}\text { Información financiera en tiempo real, gestor contable, e-Procurement, } \\
\text { presentación de impuestos, medios de pago inalámbrico (NFC, QR). }\end{array}$ \\
\hline
\end{tabular}

Beneficios tales comola cualificación de la mano de obra permiten aceptar el uso delas TIC; por ejemplo, debería haberuna reducción en los costos de las actividades de capacitación gracias al uso del e-learning o una reducción en los costos de nómina derivada de tener una mejor educación (Cervera, 1999). A esto se suma quelas habilidades necesarias para usar adecuadamente la innovación son adquiridas, a menudo, mediante instrucción informal en el trabajo, por ejemplo, mediante el adiestramiento por parte de un compañero más experimentado, mientras, simultáneamente realiza sus actividades laborales (Bayo, Billón \& Lera, 2008).

Sin embargo, la gran mayoría de las pyme solo invierten en capacitación y tecnología cuando es necesario renovar la maquinaria, no antes, debido a que este rubro no está contemplado en su plan financiero (Gomez et al., 2007).

Eluso efectivo de las TIC repercuteen una mayor productividad, mejores y más innovadores procesos, mayor calidad de los productos y servicios, reducción de costos y menores tiempos de entrega, teniendo en cuenta que no solo la inversión en computadoras es lo que hace mejorar en estos aspectos, sino la inversión en capacitación y nuevas formas de gestión que son colaterales al proceso de adquisición de nuevas tecnologías a nivel empresarial (Martin \& Omrani, 2014).

También es posible reconocer, dentro de cada proceso de la cadena de valor, las diferentes tecnologías que se pueden hallar en las empresas. Estas pueden variar deacuerdo al sector de cada una de ellas, identificando diversas herramientas que impactan de alguna manera los procesos innovadores de la organización. Asimismo, se pueden identificar las herramientas tecnológicas presentes en los procesos de la organización, clasificadas dentro de la cadena de valor, como se observan en la Tabla 2.

Fuente: Elaboración propia basada en Porter (2001). 
De todas estas tecnologías se espera que mejoren directamente el desempeño empresarial en las actividades de la cadena de valor (por ejemplo, el rendimiento del proveedor, innovaciones de producto o la prestación del servicio al cliente), ya que sus capacidades avanzadas ofrecen la promesa de una mayor integración organizativa, obteniendo unos beneficios indirectos (Xue, Ray \& Sambamurthy, 2013), aunque el efecto de las TIC en el desempeño financiero no es el mismo para todas las empresas y puede depender de las decisiones estratégicas adoptadas por cada organización (Shin, 2006).

Igualmente, antes de implementar alguna de estas tecnologías, se debe hacer un análisis completo desde lo económico, lo operacional, la adaptabilidad en los procesos de la organización y las capacidades técnicas humanas, entre otros, para que sean apropiadas adecuadamente.

\section{Conclusiones}

La innovación no es un tema nuevo, y, de acuerdo con lo mencionado por diversos autores, tiene una relación importante con el uso de las TIC en el interior de las organizaciones. Este artículo ha identificado diferentes trabajos académicos, lo que ha permitido constatar que existe un gran interés por analizar el uso de las TIC en las empresas y su impacto en los procesos de innovación de las organizaciones. Se han identificado las variables más utilizadas en los estudios que permiten relacionar las TIC con la innovación empresarial. En este sentido, se ha identificado el uso y adopción de las TIC como el elemento de mayor análisis en los estudios, reconociendo en estas tecnologías un recurso que puede direccionar a las empresas a encontrar nuevas oportunidades para ampliar su gama de productos impactando en su rendimiento empresarial.

Existe un conjunto de $\mathrm{TIC}$ dentro de la cadena de suministro (diseño asistido, simuladores, SCM, impresión 3D, gestión de redes, RFID, GPS, compra en línea, CRM, herramientas de colaboración, ERP, etc.) que pueden llegar a mejorar los procesos de innovación en las empresas; sin embargo, es necesario hacerunanálisis desdelooperacional,loeconómico (costos), la adaptabilidad en los procesos, las capacidades humanas, etc., ya que para su correcta apropiación podría ser necesario reestructurar algunos procesos.

Si la empresa desea tener un mayor impacto de las TIC sobre los procesos de innovación, se sugiere saber muy bien si las herramientas tecnológicas de las que dispone proporcionan alguna ventaja con respecto a los procesos de innovación que se desarrollan actualmente en la organización. Del mismo modo, se sugiere conocer si dichas tecnologías tienen potencial para mejorarse o si ya llegaron a su límite.

Asimismo, las empresas son cada día más sensibles a la importancia de los procesos de innovación, a través de modificaciones o mejoras de sus procesos productivos y están realizando un gran esfuerzo para poner en marcha medidas dirigidas a potenciar su desarrollo tecnológico, tratando de ser más competitivas. En este sentido, las empresas pueden encontrar en las $\mathrm{TIC}$ un recurso que favorece el progreso de estos aspectos, y generar procesos productivos para la organización.

Finalmente, bajo ciertos parámetros, las tecnologías de la información pueden proporcionar un nuevo impulso a la tendencia de innovar, llevando a las empresas a diversificar su línea de negocios, enfoque que comenzó hace décadas en los Estados Unidos (Markides, 1995), y que con las nuevas tendencias que en este campo se siguen dando, resulta un área de investigación bastante atractivo. También, futuros estudios podrían retomar esta investigación para analizar elementos como los tipos de innovación predominantes en las empresas según el tamaño y sector de actividad, ya que, en general, las investigaciones en este campo se han centrado predominantemente en los impactos.

\section{Agradecimientos}

Quisiéramos agradecer a Compensar Unipanamericana Institución Universitaria y a la empresa Draco Servicios por su apoyo y colaboración en el desarrollo de este proyecto de investigación.

\section{Referencias}

Acevedo, M. C., Moreno, S. C. \& Sánchez, H. D. (2013). Innovación empresarial: una mirada desde la competitividad, el desarrollo local y la trasformación productiva para la internalización en Colombia. Semestre Económico Universidad de Medellín, 16.

Alderete, M. \& Gutiérrez, L. (2012). TIC y productividad en las industrias de servicios en Colombia. Lecturas de Economía, 77, 163-188.

Almenara, J. C. (2004). Cambios organizativos y administrativos para incorporación de las TICs a la formación. Medidas a adoptar. Eduteca, Revista Electrónica de Tecnología Educativa, 18.

Arcila, C., Calderín, M. \& Aguaded, I. (2015). Adoption of ICTs by communication researchers for scientific diffusion and data analysis. El Profesional de la Información, 24(5), 526. doi: 10.3145/epi.2015.sep.03.

Argüello, G. M. (2015). Uso de las TIC en las PYME del sector industrial del Área Metropolitana de Bucaramanga. I+ D Revista de Investigaciones, 6(2), 125-136. https:// doi.org/10.33304/revinv.v06n2-2015009

Avendaño, W. (2012). Innovación: Un proceso necesario para las pequeñas y medianas empresas del municipio de San José de Cúcuta, Norte de Santander (Colombia). Semestre Económico, 15(31), 187-208. 
Barreto, M. L. (2017). Innovaciones de productos y financiación pública de $I+D$ : Cómo manejar la heterocedasticidad y la autocorrelación. I+ D Revista de Investigaciones, 9(1), 138-145. https://doi. org/10.33304/revinv.v09n1-2017013

Bayo, M. A., Billón, M. \& Lera, L. F. (2008). Skills, technology and organisational innovation in Spanish firms. International Journal of Manpower, 29(2), 122-145.

Breschi, S., Lissoni, F. \& Montobbio, F. (2007). The scientific productivity of academic inventors: new evidence from Italian data. Economics Innovation New Technology, 16(2), 101-118.

Cano, J. A. \& Baena, J. J. (2013). Retos en la implementación de las TIC para el proceso de negociación internacional. Cuadernos de Administración, 29(50), 153-163.

Castellanos, J., Loaiza, H. \& Cuesta, C. (2016). Importancia de las TIC para la competitividad de las Pymes en Colombia. Puente, 10(1), 93-99. http://dx.doi. org/10.18566/puente.v10n1.a10

Cervera, M. G. (1999). Las Tecnologías de la Información y la Comunicación como favorecedoras de los procesos de autoaprendizaje y de formación permanente. Educar, 25, 53-60.

Damanpour, F. \& Gopalakrishnan, S. (1998). Theories of organizational structure and innovation adoption: the role of environmental change. Journal of Engineering and Technology Management, 15(1), 1-24.

Dawid, H. \& Reimann, M. (2011). Diversification: a road to inefficiency in product innovations. Journal of Evolutionary Economics, 21(2), 191-229.

Devaraj, S. \& Kohli, R. (2003). Performance impacts of information technology: is actual usage the missing link? Management Science, 49(3), 273-289.

Espinosa, C., Reyes, L., Aponte, L., Piña, M. \& Hernández, R. (2016). Creación de una empresa que ofrezca servicios de orientación y capacitación académica.(Tesis para obtener título de grado Ingeniero de Transporte). Unidad profesional interdisciplinaria de ingeniería y ciencias sociales y administrativas.

Fonseca, D. E. (2013). Desarrollo e implementación de las TICS en las Pymes de Boyacá - Colombia. Revista FIR, Faedpyme International Review, 2.

Gálvez, A., De Lema, E. J. \& García, P. D. (2012). Impacto de la innovación sobre el rendimiento de la mipyme: Un estudio empírico en Colombia. Estudios Gerenciales, 28(122), 11-27. https://doi.org/10.1016/s01235923(12)70191-2

Gálvez, E. J. (2014). Tecnologías de información y comunicación, e innovación en las MIPYMES de Colombia. Cuadernos de Administración, 30(51).

Garcia, V. M. (2007). Does technological diversification promote innovation?: An empirical analysis for European firms. Research policy, 35(2), 230-246.
García, O. A., López, J. I. \& Epalde, J. G. (2015). Factores que afectan la relación entre diversificación y rendimiento. Revisión de la literatura. Dirección y Organización, (57), 4-18.

Gomez, S. A., Gonzalez, L. J., Lusa, B. E. \& Osorio, M. J. L. (2007). Innovación y nuevas tecnologías en las Pymes de Galicia, Los sectores Textil y Conservero. Revista Galega de Economía, 15.

Graells, P. M. (2000). Las TIC y sus aportaciones a la sociedad. Departamento de pedagogía aplicada.

Hernández, A., Marulanda, C. E. \& López, M. (2014). Análisis de capacidades de gestión del conocimiento para la competitividad de Pymes en Colombia. Información tecnológica, 25(2), 111-122. https://doi.org/10.4067/ s0718-07642014000200013

Hoyos, C. (1999). Un modelo para investigación documental: guía teórico-práctica sobre construcción de Estados del Arte con importantes reflexiones sobre la investigación: Señal Editora.

Hoyos, C. J. A. \& Valencia, A. A. (2012). El papel de las TIC en el entorno organizacional de las Pymes. Trilogía, 7, $105-122$.

Jiménez, Z. A. I., Martínez, R. M. P. \& Barba, S. V. (2009). Cooperación y tic como factores de éxito en la innovación en productos: un análisis empírico para la pymes españolas. Revista da Micro e Pequena Empresa, Campo Limpo Paulista, 3(2), 132-150.

Jimenez, Z. A. I. \& Torrent, S. J. (2009). Orientación proactiva hacia el cliente, cooperación y uso de las TIC: un análisis empírico sobre sus interrelaciones y efectos como potenciadores de la innovación en producto. INNOVAR, 19(33), 22.

Jones, C. \& Morero, H. A. (2014). Factores explicativos de la adopción de las TIC en las tramas productivas automotriz y siderúrgica de Argentina. Pensamiento y gestión, 37.

Kahn, K. B. K. A. (2001). Market Orientation, Interdepartmental Integration, and Product Development Performance. Journal of Product Innovation Management 18, 314-323.

Kim, H. D., Lee, I. \& Lee, C. K. (2013). Building Web 2.0 enterprises: A study of small and medium enterprises in the United States. International Small Business Journal, 31(2), 156-174.

López, G. C. \& Robledo, V. J. (2014). Una aproximación a la gestión de capacidades de innovación en la pyme colombiana. Gestión \& Sociedad, 7(2), 11-30.

Manual de Oslo- Guía para la recogida e interpretación de datos sobre innovación. (2005).

Markides, C. C. (1995). Diversification, restructuring and economic performance. Strategic Management Journal, 16(2), 101-118.

Martin, L. \& Omrani, N. (2014). An assessment of trends in technology use, innovative work practices and 
employees' attitudes in Europe. Applied Economics, 47(6), 623-638. https://doi.org/10.1080/00036846.2 014.978072

Martínez, A. \& Castillo, A. E. (2014). Comunicación Institucional en España. Estudio del uso que los diputados españoles hacen de las TIC en sus relaciones con la ciudadanía. Revista Latinoamericana de Comunicación, 126.

Marulanda, C. \& López, M. T. (2013). La gestión del conocimiento en las Pymes de Colombia. Revista Universidad católica del norte, 38, 1-13.

Marulanda, C. E., Giraldo, J. \& López, M. (2014). Acceso y uso de las Tecnologías de la información y las Comunicaciones (TICs) en el aprendizaje: El Caso de los Jóvenes Preuniversitarios en Caldas, Colombia. Formación universitaria, 7(4), 47-56. doi: 10.4067/ s0718-50062014000400006.

McDonough, E. F., Kahnb, K. B. \& Barczaka, G. (2001). An investigation of the use of global, virtual, and colocated new product development teams. Journal of product innovation management, 18(2), 110-120.

Miller, D. J., Fern, M. J. \& Cardinal, L. B. (2007). The use of knowledge for technological innovation within diversified firms. Academy of Management journal, 50(2), 308-326.

Mosquera, W., Rojas, L. \& Grillo, C. (2015). Relación entre el uso efectivo de las TIC y las decisiones gerenciales en Pymes. Revista de la Facultad de Ciencias Químicas, 12.

Osorio, G. C., Londoño, M. J. \& López, Z. E. (2016). Analysis of factors that influence the ICT adoption by smes in Colombia. Intangible Capital, 12(2), 666. doi: 10.3926/ ic.726.

Porter, M. E. (2001). Strategy and the Internet. Harvard Business Review, 79(3), 62-79.

Riascos, E., Aguilera, C. \& Solano, R. (2015). Efectividad de las TIC en los procesos administrativos de las Pymes de Santiago de Cali - Colombia. Revista GTI, 14(40), 17-30.

Rothwell, R. (1994). Towards the fifth-generation innovation process. International marketing review, 11(1), 7-31.

Salvador, V. J., Brand, G. V. \& Salazar, C. G. (2011). La innovación y la utilización de las TICS como bases de crecimiento de las Pyme (Caso Aguascalientes México). I Congreso online sobre Los Modelos Latinoamericanos de Desarrollo.

Schumpeter, J. (1934). Capitalism, socialism, and democracy. New York: Harper \& Row.

Segovia, J. G., Hernández, R. M., \& Ramírez, A. R. (2013). Innovación y conocimiento tecnológico en la sociedad del siglo XXI: la revolución de las TIC S. Derecom, (13), 7.

Sharma, R. (2013). An Appraisal of Internet Business
Models as a Research Paradigm Innovation in the High-Tech Economy (pp. 35-51): Springer.

Shin, N. (2006). The impact of information technology on the financial performance of diversified firms. Decision Support Systems, 41(4), 698-707. https://doi. org/10.1016/j.dss.2004.10.003

Smith, P. G. \& Blanck, E. L. (2002). From experience: leading dispersed teams. Journal of Product Innovation Management, 19(4), 294-304.

Soto, C. F., Senra, A. I. \& Neira, M. C. (2009). Ventajas del uso de las TICS en el proceso de enseñanza-aprendizaje desde la óptica de los docentes universitarios españoles. Edutec. Revista electrónica de Tecnología educativa, 29.

Soto, I. O. \& Rios, C. P. P. (2017). Comportamiento y experiencia de consumo desde la interconexión e interactividad de la World Wide Web: un recorrido teórico. I+ D Revista de Investigaciones, 8(2).

Triana, J., Medina, V. \& Rodríguez, J. (2013). Modelo para fortalecer el rol de las pymes en el emprendimiento de Bogotá D. C. Revista Científica, 17.

Tushman, M. \& Nadler, D. (1986). Organizing for innovation. California management review, 28(3), 7492.

Urquía, G. E. \& Pérez, E. (2012). Percepción y uso de las TIC en la contabilidad de las Pymes: evidencia empírica en España: Universidad Complutense de Madrid.

Vilaseca, R. J., Torrent, S. J., Lladós, M. J. \& Garay, T. (2007). Tecnologías de la información y la comunicación, innovación y actividad turística: hacia la empresa en red. Cuadernos de Turismo, (19).

Wiersema, M. F. \& Bowen, H. P. (2008). Corporate diversification: The impact of foreign competition, industry globalization, and product diversification. Strategic Management Journal, 29(2), 115-132. https://doi.org/10.1002/smj.653.

Xue, L., Ray, G.\& Sambamurthy, V. (2013). The impact of supply-side electronic integration on customer service performance. Journal of Operations Management, 31(6), 363-375.

Zhang, X., van Donk, D. P. \& van der Vaart, T. (2011). Does ICT influence supply chain management and performance?: A review of survey-based research. International Journal of Operations and Production Management, 31(11), 1215-1247. 